\title{
Efecto del porcentaje de pildorado sobre la implantación y productividad de alfalfa (Medicago sativa $\mathrm{L}$. )
}

Odorizzi, A.S.; V. Arolfo, D.H. Basigalup y S. Solá

\begin{abstract}
RESUMEN
El pildorado consiste en revestir a la semilla de alfalfa con una mezcla de rizobios, un fungicida o un insecticida, carbonato de calcio y un aglutinante. El objetivo fue comparar porcentajes de pildorado (10\%, 20\%, 30\%, $50 \%$ y $75 \%$ ) y semilla desnuda, con respecto a la densidad de plantas establecidas y la producción forrajera. Se implantó un ensayo con tres repeticiones en otoño de 2013 con una densidad fija (10 kg por ha), y se analizó el número de plantas por $\mathrm{m}^{2}$ a diferentes días de la siembra, y al finalizar la primera temporada y la producción forrajera. Hasta los 17 y 29 días las plantas por $\mathrm{m}^{2}$ fueron superiores en los dos tratamientos con semilla desnuda. A partir de los 46 días, los tratamientos con menor porcentaje de pildorado se ubicaron entre los mayores valores de plantas por $\mathrm{m}^{2}$. Los niveles altos de pildorado (30, 50 y $75 \%)$ presentaron la menor cantidad de plantas por $\mathrm{m}^{2}$. Las mayores producciones de forraje se obtuvieron con bajos porcentajes de pildorado y con semilla desnuda. Las semillas con $75 \%$ de pildorado tuvieron el menor rendimiento acumulado de forraje y uno de los menores valores de número de plantas por $\mathrm{m}^{2}$ al final de la temporada.
\end{abstract}

Palabras clave: rizobios, leguminosas, peleteado.

Odorizzi, A.S.; V. Arolfo, D.H. Basigalup and S. Solá 2017. Effect of seed coating percentage on alfalfa (Medicago sativa L.) Implantation and forage yield. Agriscientia 34: 71-77

\section{SUMMARY}

Alfalfa seed coating consists of coating the seed with a mixture of rhizobia, a fungicide or an insecticide, calcium carbonate and a binding substance. The aim of the study was to compare seed coat percentages (10\%, $20 \%, 30 \%, 50 \%$ and $75 \%$ ) and naked seed in relation to established plants and forage yield. Following a design with 3 reps, a trial was established in 2013 at $10 \mathrm{~kg}$ per ha in which the number of plants per $\mathrm{m}^{2}$ was studied by counting at different days of sowing and at the end of the first season and forage yield. Up to 17 and 29 days after sowing the number of plants per $\mathrm{m}^{2}$ was higher in the two naked seed treatments. After 46 days of sowing, the treatments with the lowest seed coating percentage showed the highest numbers of plants per $\mathrm{m}^{2}$. High seed coating percentages (30\%, 50\% and $75 \%$ ) had the lowest number of plants per 
$\mathrm{m}^{2}$. The highest forage yields were obtained with low seed coating percentages and naked seed treatments. By contrast, seeds with $75 \%$ coating treatment exhibited the lowest accumulated forage yield and one of the lowest values of number of plants per $\mathrm{m}^{2}$ at the end of the season.

Key words: rhizobia, leguminous, pelleting.

A.S Odorizzi, V. Arolfo y D.H. Basigalup. INTA - EEA Manfredi. Mejoramiento Genético de Alfalfa. Ruta Nac. No 9 km. 636, 5988, Manfredi, Córdoba, Argentina. Tel: (54) 3572-493053, fax: (54) 3572-49306. S. Solá. Rizobacter. Jefe de investigación y calidad de semillas. Avda. Dr. Arturo Frondizi No 1150, Parque Industrial - C.P. B2702HDA, Pergamino (Bs.As.), Argentina. Correspondencia a: odorizzi.ariel@inta.gob.ar

\section{INTRODUCCIÓN}

El pildorado de la alfalfa (Medicago sativa L.), también conocido como peleteado o peletizado, es una técnica que consiste en revestir a la semilla con una mezcla de rizobios (Sinorhizobium meliloti), un fungicida y/o un insecticida, una cubierta de carbonato de calcio (material inerte) y una sustancia aglutinante que mantiene unida la mezcla y le da consistencia (Heritage Seeds, 2016).

Originalmente, el pildorado de semillas se propuso como una alternativa para incrementar la disponibilidad de rizobios y mejorar así la nodulación en las raíces de las plantas (Talley, 2010). El Natural Resources Conservation Service (NRCS, 2010) señala que el peletizado favorece significativamente la supervivencia de los rizobios, a punto tal que se registraron valores de 300 bacterias por semilla al cabo de 12 meses de almacenamiento en condiciones adecuadas. Si las condiciones de almacenado no son apropiadas (alta humedad y temperatura elevada), los rizobios y las semillas pierden viabilidad en forma significativa aun estando peletizadas. En consecuencia, es muy importante mantener la semilla pildorada en lugares frescos y secos. En caso de duda sobre la viabilidad de los rizobios, se aconseja añadir inoculante fresco a las semillas (Murphy et al., 1984).

En aquellos casos en que la siembra y la aplicación de fertilizantes se hagan en un solo paso, el pildorado de las semillas puede proteger a los rizobios contra el daño que pueden causarles los fertilizantes granulados (Leep et al., 2012).

Por otra parte, el agregado de fungicidas pro- porciona protección a la plántula contra enfermedades de hongos de suelo, principalmente damping off, causada por Pythium spp. y/o Phytophtora megasperma f. sp. medicaginis. Este efecto es particularmente útil cuando las condiciones ambientales (alta humedad y temperaturas moderadas a bajas) favorecen el ataque de esos patógenos a las plántulas. El agregado de insecticidas otorga protección temporaria contra insectos de suelo, pulgones y trips, plagas que pueden ocasionar severos daños en las etapas de emergencia e implantación del cultivo (Aragón \& Inwinkelried, 2007).

Otro efecto beneficioso del peleteado es que el revestimiento de carbonato de calcio afecta el $\mathrm{pH}$ alrededor de la semilla; de esta manera contrarresta la acidez propia que pudiera tener el suelo o la causada por el fertilizante cuando se aplica simultáneamente en la siembra (Heritage Seeds, 2016).

Según Talley (2012), el pildorado debe centrarse en maximizar el número de plantas sanas establecidas por cada kilogramo de semilla sembrado. Esto va en consonancia con lo señalado por Twidwell \& Gallenberg (2002), quienes establecieron que el peletizado tiene por objeto aumentar la densidad de plantas en comparación con la de semillas no peletizadas, a través de una tasa más alta de supervivencia de plántulas. En este contexto, si se usara semilla peletizada, se necesitarían menos semillas por $\mathrm{m}^{2}$ para establecer una adecuada densidad de plantas en la pastura que con la siembra de semilla no peletizada. De acuerdo con Leep et al. (2012), para obtener un número determinado de plantas establecidas en la pastura el peletizado permitiría utilizar hasta un 33\% menos de semilla 
que la siembra de semilla no peletizada.

Respecto de la semilla desnuda, el pildorado aumenta el peso de la semilla en un porcentaje variable según la cantidad de material inerte que se utilice. Algunas recomendaciones indican como deseable un incremento de peso de hasta un tercio del peso de la semilla no recubierta (Leep et al., 2012). Por ello, con la semilla peletizada, se debe prestar atención a la densidad real de siembra que se está generando, dado que el número inicial de plantas por unidad de superficie se relaciona directamente con la cantidad de semilla sembrada.

Desde hace varios años, casi la totalidad de la semilla de alfalfa que se comercializa en Argentina está peleteada (Basigalup \& Rossanigo, 2007). Sin embargo, los beneficios de esta técnica pueden verse neutralizados, e incluso eliminados, por un incremento excesivo en el peso del pildorado, lo que equivaldría a sembrar menor cantidad de semillas afectando significativamente la población de plantas del alfalfar. Esto es particularmente importante porque el productor usualmente no corrige la densidad de siembra en función del pildorado, sino que tiende a utilizar una cantidad fija de kg por ha, independientemente del tratamiento que tenga la semilla. Además, dado que algunas empresas semilleras incrementan el peso por peleteo por encima del $50 \%$, la cantidad real de semillas por unidad de superficie podría ser mucho menor a la recomendada.

El objetivo de este trabajo fue evaluar el efecto de la cantidad de pildorado agregado en semillas de alfalfa sobre la cantidad efectiva de semilla sembrada, la densidad de plantas establecidas y la producción de forraje de un alfalfar sembrado a una densidad de siembra fija ( $k g$ por ha sembrados, ya sea que se siembre semilla pildorada o semilla desnuda).

\section{MATERIALES Y MÉTODOS}

Las evaluaciones planteadas en este trabajo se desarrollaron en el campo experimental y en los laboratorios de la Estación Experimental Agropecuaria (E.E.A.) del Instituto Nacional de Tecnología Agropecuaria (INTA) Manfredi, ubicada a los $31^{\circ}$ 49' $12^{\prime \prime}$ S, $63^{\circ} 46^{\prime} 00^{\prime \prime}$ O y 292 m.s.n.m. durante las temporadas productivas 2013/2014 y 2014/2015. El clima de la EEA Manfredi se caracteriza por ser templado, con una precipitación media anual de 760 mm, distribuidos según un régimen de tipo monzónico. El suelo está clasificado como Haplustol éntico (Serie Oncativo), profundo, bien drenado, desarrollado sobre materiales loéssicos de textura franco-limosa que ocupan las lomas casi planas y muy extendidas. La secuencia de horizontes encontrada comúnmente es $\mathrm{A}, \mathrm{AC}$ y Ck; los dos primeros presentan estructuras de bloques subangulares, y el tercero presenta estructura masiva (INTA - SMAGyRR, 1987).

El ensayo fue sembrado en marzo de 2013, con una densidad fija (tanto de semilla viable desnuda como semilla viable + diferentes porcentajes de pildorado) de $10 \mathrm{~kg}$ por ha. Siguiendo un diseño en bloques completos aleatorizados (BCA) con tres replicaciones y parcelas de $1 \times 5 \mathrm{~m}$. Se compararon siete tratamientos $(\mathrm{T}): \mathrm{T} 1=10 \%$ pildorado; $\mathrm{T} 2=20 \%$ pildorado; $\mathrm{T} 3=30 \%$ pildorado; $\mathrm{T} 4=50 \%$ pildorado; $\mathrm{T} 5=75 \%$ pildorado; T6 = semilla desnuda tratada con fungicida e insecticida y $T 7$ (control) = semilla desnuda.

Los tratamientos de peletizado estuvieron conformados por: fungicida + insecticida + rizobios + cantidades variables de material inerte. El fungicida sistémico, diseñado para ser aplicado a las semillas fue Apron Gold $®$ cuyo ingrediente activo es el metalaxil-M, perteneciente a la clase química de las fenilamidas. El insecticida de amplio espectro y acción residual, con actividad sistémica radicular utilizado fue Cruiser $60 ®$ cuyo ingrediente activo es el tiametoxam. Los tratamientos de pildorado incluyeron la inoculación con $S$. meliloti cepa B399. Mediante carbonato de calcio como material inerte se llegó al porcentaje estipulado en cada tratamiento de pildorado. El pildorado no afectó el poder germinativo de las semillas. El poder germinativo, tanto al principio como después del pildorado fue en promedio del $93 \%$ lo que indicaría que los tratamientos se hicieron de acuerdo a los parámetros de calidad industrial definidos como adecuados.

Para evaluar el efecto de los tratamientos de pildorado sobre las semillas y sobre la densidad de plantas establecidas, se realizaron las siguientes determinaciones: a) peso de 1000 semillas (P1000), de acuerdo a las normas definidas por la International Seed Testing Association (ISTA, 2007), antes y después de los tratamientos (T1 a T6); b) porcentaje de material inerte de las 1000 semillas para los tratamientos 1 a 6; c) evolución del número de plantas por $\mathrm{m}^{2}$ mediante el uso de un aro de $1 / 4 \mathrm{~m}^{2}$ colocado en una estación fija y representativa de cada parcela y efectuando conteos a los 17 (C1), 29 (C2), 46 (C3) y 204 (C4) días de la siembra y a la finalización (C5) de la primera temporada de evaluación. Para el cálculo del porcentaje de material inerte en cada tratamiento, primero se pesaron 1000 semillas pildoradas (PSP); luego se lavó con agua todo el recubrimiento, se secaron en estufa a $60{ }^{\circ} \mathrm{C}$ hasta peso constante con el fin de secar superficialmente y se pesaron 
las 1000 semillas desnudas (PSD). La estimación se realizó mediante la fórmula:

\section{Procentaje de material inerte $(\%)=$} (PSP - PSD / PSP) $\times 100$

Las determinaciones de producción de forraje se realizaron mediante cortes al 10\% de floración o cuando los rebrotes alcanzaron los $5 \mathrm{~cm}$ de altura desde la corona. Se cosechó mediante maquinaria autopropulsada de corte y se pesó todo el forraje de cada parcela obtenido con cortes a una altura de $5 \mathrm{~cm}$. La producción anual acumulada (Racum) de cada tratamiento se expresó como la suma de los cortes en $\mathrm{kg}$ de materia seca por hectárea por temporada ( $\mathrm{kg} \mathrm{MS} \mathrm{ha-1}$ temporada $\left.^{-1}\right)$. Se realizaron seis cortes durante la temporada 2013/2014 (08/10/2013, 14/11/2013, 11/12/2013, 09/01/2014, $13 / 02 / 2014$ y $02 / 04 / 2014$ ) y cinco cortes durante la temporada 2014/2015 (10/10/2014, 04/11/2014, 05/12/2014, 05/01/2015 y 06/02/2015). Las determinaciones del porcentaje de materia seca parcial (\% MS) en cada corte se hicieron sobre muestras compuestas de $200 \mathrm{~g}$ por parcela, pesadas en húmedo, secadas en estufa a $60^{\circ} \mathrm{C}$ hasta peso constante y vueltas a pesar.

Todos los datos se analizaron estadísticamente con el software Infostat (Balzarini y Di Rienzo, 2011). Se realizó un análisis de la varianza para cada una de las variables estimadas y las medias se contrastaron mediante la prueba de comparaciones múltiples $(p<0,05)$ DGC (Di Rienzo et al., 2002).

\section{RESULTADOS Y DISCUSIÓN}

\section{Peso de 1000 semillas}

El análisis de varianza permitió detectar diferencias $(p<0,05)$ entre los tratamientos para el P1000. La Figura 1 muestra el resultado de la prueba de comparaciones múltiples DGC entre las medias de los tratamientos. El P1000 se incrementó $(p<0,05)$ en relación directa con el aumento del porcentaje de pildorado, mientras que los tratamientos control (6 y 7), sin recubierta de material inerte, exhibieron los valores más bajos.

\section{Porcentaje de material inerte}

En la Figura 2 se observa el resultado de la prueba de comparaciones múltiples DGC entre las medias de los tratamientos. El porcentaje de material inerte se incrementó $(p<0,05)$ en relación

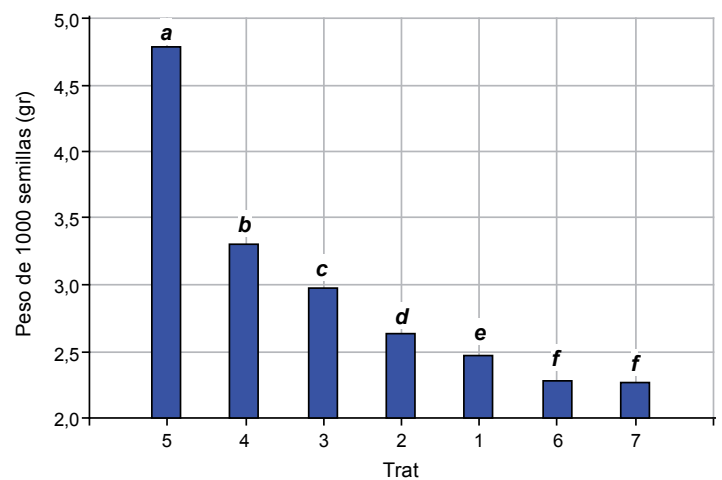

Figura 1. Comparaciones múltiples de medias de peso de 1000 semillas entre semillas desnudas ( $T 6=$ semilla desnuda tratada con fungicida e insecticida y $\mathrm{T} 7=$ semilla desnuda) y con porcentaje creciente de pildorado ( $\mathrm{T} 1=10 \%$ pildorado; $\mathrm{T} 2=20 \%$ pildorado; $\mathrm{T} 3=30 \%$ pildorado; $\mathrm{T} 4=50 \%$ pildorado; $\mathrm{T} 5=75 \%$ pildorado). Trat $=$ tratamientos. Test: $D G C$ Alfa $=0,05$. Medias con la misma letra no son significativamente diferentes $(p<0,05)$.

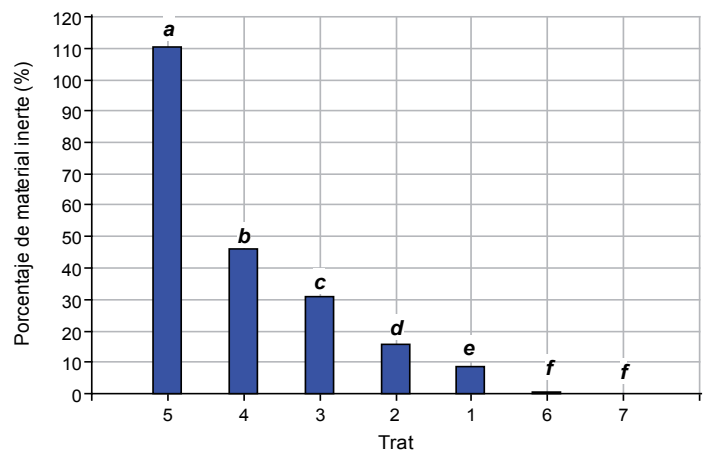

Figura 2. Comparaciones múltiples del porcentaje de inerte entre semillas desnudas ( $T 6=$ semilla desnuda tratada con fungicida e insecticida y $\mathrm{T} 7=$ semilla desnuda) y con porcentaje creciente de pildorado ( $\mathrm{T} 1=10 \%$ pildorado; $\mathrm{T} 2=20 \%$ pildorado; $\mathrm{T} 3=30 \%$ pildorado; $\mathrm{T} 4=50 \%$ pildorado; $\mathrm{T} 5=75 \%$ pildorado). Trat $=$ tratamientos. Test: DGC Alfa=0,05. Medias con la misma letra no son significativamente diferentes $(p<0,05)$.

directa con el incremento del pildorado, con la excepción de los tratamientos 6 y 7 (no pildorados). Es interesante destacar que el tratamiento 5 registró un incremento del porcentaje de material inerte $(110 \%)$ por encima del valor del pildorado (75\%).

\section{Densidad de plantas}

La evolución del número de plantas por $\mathrm{m}^{2}$ a lo largo de las fechas de conteo se presenta en la Figura 3.

Los dos tratamientos de semilla desnuda presentaron mayor cantidad de plantas establecidas que los tratamientos pildorados, aunque las dife- 


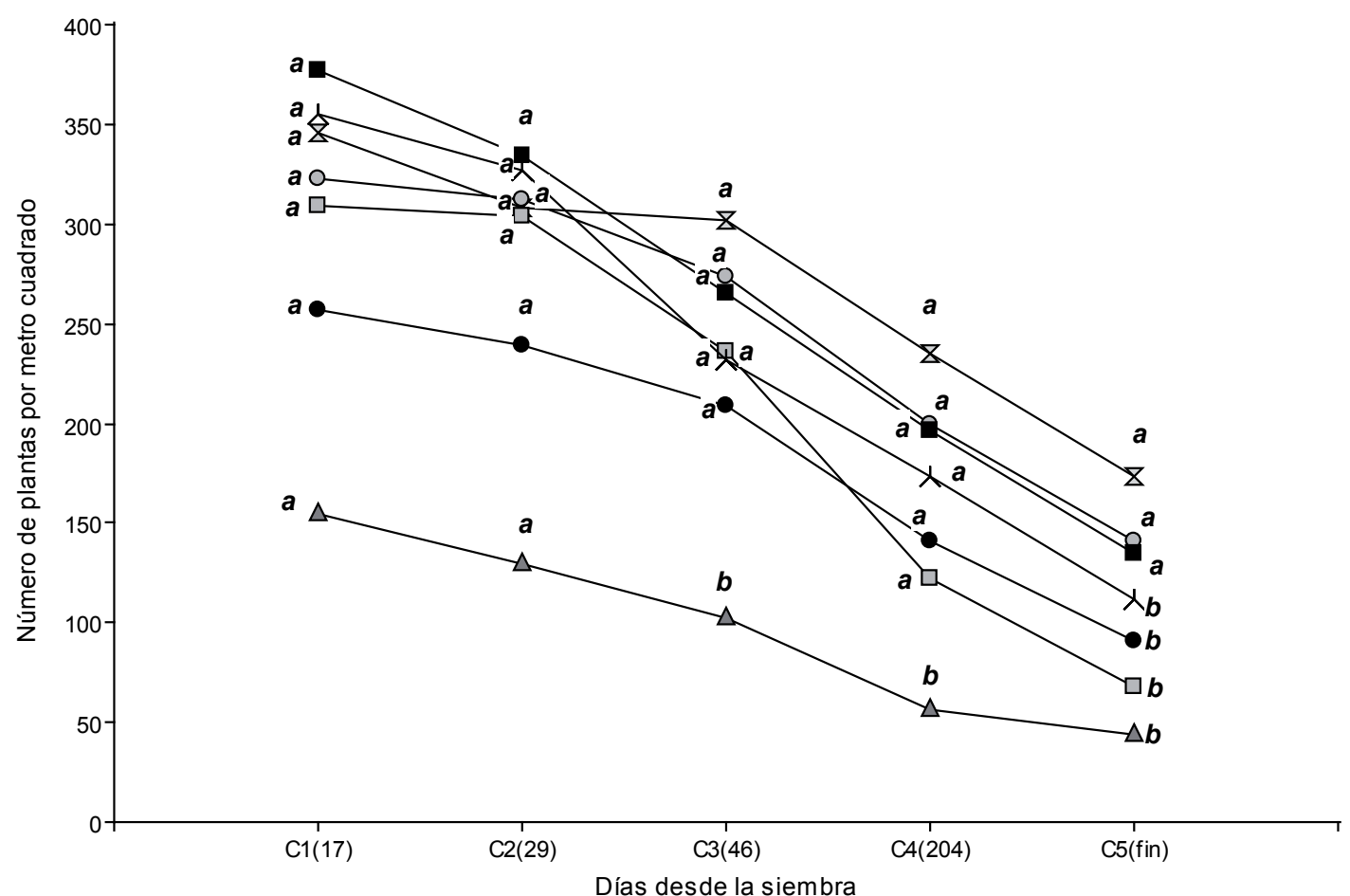

\begin{tabular}{|c|c|c|c|}
\hline$-\nabla-10 \%$ & $-0-20 \%$ & $\longrightarrow-30 \%$ & $\square-50 \%$ \\
\hline$-\triangle-75 \%$ & $\longrightarrow$ Solo curada & ᄃ Testigo & \\
\hline
\end{tabular}

Figura 3. Evolución del número de plantas por $\mathrm{m}^{2}$ para los tratamientos de semilla $\mathrm{T} 1=10 \%$ pildorado, $\mathrm{T} 2=20 \%$ pildorado, $\mathrm{T} 3=30 \%$ pildorado, $\mathrm{T} 4=50 \%$ pildorado, $\mathrm{T} 5=75 \%$ pildorado, $\mathrm{T} 6=$ semilla desnuda tratada con fungicida e insecticida y $\mathrm{T} 7=$ semilla desnuda a lo largo de la primera temporada de evaluación (2013/14). Referencias: conteos a los 17 (C1), 29 (C2), 46 (C3) y 204 (C4) días de la siembra y a la finalización (C5) de la primera temporada de evaluación.

rencias no fueron significativas en los dos primeros muestreos a los 17 y 29 días de la siembra (Figura 3). Esto se atribuye a que a igual densidad de siembra ( $\mathrm{kg}$ semilla desnuda o semilla + semilla con pildorado por ha) la cantidad efectiva de semillas depositadas por unidad de superficie fue mayor en los tratamientos sin pildorado que en aquellos con pildorado. A partir de los 46 días y con el cultivo ya establecido (204 días de la siembra) el tratamiento con $75 \%$ de pildorado presentó un número de plantas por $\mathrm{m}^{2}$ significativamente menor con respecto a los tratamientos restantes. Al final de la primera temporada productiva, el tratamiento 1 ( $10 \%$ de pildorado), 2 ( $20 \%$ de pildorado) y 6 (semilla desnuda tratada con fungicida e insecticida) alcanzaron los mayores números de plantas por $\mathrm{m}^{2}$, sin diferir significativamente entre ellos aunque el tratamiento 1 mostró un valor numéricamente superior a los tratamientos 2 y 6 (Figura 3). Los tratamientos con semilla desnuda y los restantes tratamientos se ubicaron con valores intermedios o menores en cantidad de plantas por $\mathrm{m}^{2} \mathrm{sin}$ diferencias estadísticamente significativas en ellos. Es interesante señalar que el tratamiento 6 ofreció siempre valores más altos de densidad de plantas que el tratamiento con semilla desnuda o control (T7). Esto podría indicar un efecto beneficioso de los tratamientos con fungicida e insecticida que pudieron disminuir el daño provocado por insectos y/u hongos de suelo durante el establecimiento de las plantas. Una situación similar fue informada por Twidwell \& Gallenberg (2002), quienes en un ensayo donde compararon tres tratamientos (semilla desnuda, semilla desnuda tratada con fungicida y semilla pildorada al 33\%) sembrados con una densidad fija de $15 \mathrm{~kg}$ por ha, determinaron que la semilla tratada sólo con fungicida proporcionó la mayor densidad de plantas, en tanto que el tratamiento con semilla desnuda registró el menor valor. De todos modos, esas diferencias en densidad de 
plantas no se reflejaron en los rendimientos de forraje, que no exhibieron diferencias significativas entre tratamientos. Por el contrario, Shewmaker et al. (2002) compararon la producción de forraje de tres variedades de alfalfa sembradas con semillas desnudas pre-inoculadas con rizobios y semillas pildoradas (33\% de incremento con yeso como material inerte + rizobios + molibdeno) y a una densidad fija de $16 \mathrm{~kg}$ por ha y detectaron diferencias significativas en la producción de MS. Independientemente de la variedad, la semilla peleteada proporciona los mayores rendimientos de forraje cuando se la compara con tratamientos preinoculados.

En el presente trabajo, los niveles altos de pildorado (30, 50 y $75 \%$ ) arrojaron las menores cantidades de plantas por $\mathrm{m}^{2}$, tanto después del primer corte como al final de la primera temporada, aunque las diferencias con los restantes tratamientos fueron significativas a los 46, 204 días y al final de la primera temporada evaluada. En el caso del tratamiento 5 , que tuvo un $75 \%$ de pildorado y esto significó un incremento de peso de la semilla del orden del $110 \%$ (Figura 2), se advierte que al haberse sembrado $10 \mathrm{~kg}$ por ha de semilla peleteada se depositaron en realidad $4,7 \mathrm{~kg}$ de semilla desnuda, valor que se encuentra muy por debajo del recomendado para lograr un buen stand de plantas. La densidad de siembra recomendada para un cultivo de alfalfa puro es de 10 a $12 \mathrm{~kg}$ por ha de semilla desnuda (teniendo en cuenta un poder germinativo del $95 \%$ y una pureza del $99 \%$ que daría un valor cultural de $94,05 \%$ y el peso de 1000 semillas de 2,5 gramos) lo que equivale a depositar entre 370 y 450 semillas por $\mathrm{m}^{2}$. No obstante, cuando se utiliza semilla pildorada sin ajustar la densidad de siembra, la pastura podría tener una cantidad inicial de plantas inferior a la recomendada (Duarte, 2007; Romero, 2008). La densidad de plantas necesaria para obtener altos rendimientos, es de aproximadamente 200 plantas por $\mathrm{m}^{2}$ en el año de establecimiento, cifra que suele disminuir entre el segundo y tercer año a 100 plantas por $\mathrm{m}^{2}$ (Parga, 1994)

\section{Producción forrajera}

Las medias de producción acumulada de forraje ( $\mathrm{kg} \mathrm{MS} \mathrm{ha}^{-1}$ ) por temporada (seis cortes en $2013 / 2014$ y cinco cortes en 2014/2015) y la producción acumulada total (ambas temporadas) para cada tratamiento, se resumen en la Tabla 1.
Tabla 1. Producción acumulada de forraje en la primera temporada (2013/2014), segunda temporada (2014/2015) y acumulada total para cada tratamiento, pildorado (1 a 5), semilla desnuda curada (6) y testigo (7)

\begin{tabular}{lrrr}
\hline Tratamiento & $\begin{array}{r}2013 / 2014 \\
\mathrm{~kg} \mathrm{MS} \mathrm{ha}^{-1}\end{array}$ & $\begin{array}{r}2014 / 2015 \\
\mathrm{~kg} \mathrm{MS} \mathrm{ha}^{-1}\end{array}$ & $\begin{array}{r}2013 / 2015 \\
\mathrm{~kg} \mathrm{MS} \mathrm{ha}^{-1}\end{array}$ \\
\hline $1(10 \%)$ & 13870,80 & 5676,83 & $19547,63 \mathrm{a}$ \\
$2(20 \%)$ & 13615,07 & 5313,28 & $18928,35 \mathrm{a}$ \\
7 (Testigo) & 13526,28 & 5371,89 & $18898,17 \mathrm{a}$ \\
6 (Solo & 12812,52 & 5304,55 & $18117,07 \mathrm{a}$ \\
curada) & 12269,15 & 4691,76 & $16960,91 \mathrm{a}$ \\
$4(50 \%)$ & 11422,46 & 4278,11 & $15700,57 \mathrm{a}$ \\
$3(30 \%)$ & 9620,15 & 3668,11 & $13288,26 \mathrm{~b}$ \\
\hline (75\%) & & & \\
\hline
\end{tabular}

El análisis estadístico detectó diferencias significativas $(p<0,05)$ entre tratamientos cuando se comparó la producción forrajera acumulada de ambas temporadas (2013/2015). Los tratamientos con menor pildorado ( 1 y 2 ) y aquellos tratamientos con semilla desnuda ( 6 y 7 ) fueron númericamente los de mayor producción forrajera, aunque no diferentes estadísticamente, para cada una de las temporadas de muestreo; el tratamiento 5 (75\% de pildorado) fue el que exhibió los menores $(p<0,05)$ promedios de rendimiento acumulado de forraje y uno de los menores conteos de plantas $\mathrm{m}^{-2}$ al final de la temporada. En general, el ordenamiento de los tratamientos en función del rendimiento de forraje guarda relación con la densidad de plantas de las parcelas (Figura 3).

Los valores de la primera temporada son los esperables para el comportamiento productivo de la alfalfa en la región. Por el contrario, los inusualmente bajos valores alcanzados durante la segunda temporada se relacionan con la deficiencia hídrica registrada en 2014/2015 y los suelos manchoneados con distinta capacidad de retención de agua (Arolfo \& Odorizzi, 2014).

De todo lo anterior, se infiere que el pildorado de alfalfa -cuando se efectúa adecuadamente- puede ser una práctica que mejore la implantación del cultivo y redunde en mayor producción forrajera. Sin embargo, cuando los porcentajes de incremento de peso de sustancias (material inerte) agregadas a la semilla son excesivos, puede tener efectos perjudiciales. Se advierte que -atentos a que la casi totalidad de semilla comercial se ofrece peleteada- las densidades de siembra recomendadas en Argentina (8-12 kg de semilla desnuda por ha) podrían ser excesivamente bajas si no se ajustaran los kilos a sembrar en función del porcentaje de incremento de peso de las semillas. Esto es particularmente importante cuando se utiliza semilla que ha sido peleteada con agregado de inerte muy al- 
tos, como lo prueban en este trabajo los tratamientos $5(75 \%), 4(50 \%)$ y $3(30 \%)$. En este contexto, se sugiere enfáticamente a los productores ajustar la densidad de siembra en función del porcentaje de pildorado. Si este último dato no estuviere disponible en la bolsa de semilla peleteada, se recomienda aumentar la dosis de siembra.

\section{BIBLIOGRAFÍA}

Aragón, J. y J. Inwinkelried, 2007. Manejo integrado de plagas de la alfalfa. En: Basigalup, D. (Ed.) El cultivo de la alfalfa en la Argentina. Ediciones INTA, Argentina. pp. 176-190.

Arolfo, V. y A. Odorizzi, 2014. Avances en alfalfa. Ensayos territoriales. Red de evaluación de cultivares de alfalfa. Año 24, № 24. Ediciones INTA. Argentina. 63 pp.

Balzarini, M.G. y J.A. Di Rienzo, 2011. InfoGen versión 2011. FCA, Universidad Nacional de Córdoba, Argentina. Disponible en: http://www.info-gen.com.ar.

Basigalup, D.H. y R. Rossanigo, 2007. Panorama actual de la alfalfa en la Argentina. En: Basigalup, D. (Ed.) El cultivo de la alfalfa en la Argentina. Ediciones INTA, Argentina. pp. 15-17.

Di Rienzo, J.A.; A.W. Guzmán and F. Casanoves, 2002. A multiple-comparisons method based on the distribution of the root node distance of a binary tree. Journal of Agricultural, Biological and Environmental Statistics, 7 (2): 129-142.

Duarte, G., 2007. Fertilización de la alfalfa (en línea). (Disponible en: http://www. produccion-animal.com.ar/ produccion_y_manejo_pasturas/pasturas_cultivadas_ alfalfa/53-fertilizacion.pdf. Consultado: 12/08/2016)

Heritage Seeds, 2016. (Disponible en: http://www.heritageseeds.com.au/research-development/agronomicadvice/advanced-seed-coating-technology Consultado: 12/08/2016).

INTA - SMAGyRR, 1987. Carta de suelos de la República Argentina. Hoja Oncativo. Córdoba. Instituto Nacional de Tecnología Agropecuaria - Secretaria Ministerio de Agricultura Ganadería y Recursos Renovables.

ISTA, 2007. International Rules for Seed Testing. International Seed Testing Association, Bassersdorf, Suiza.

Leep, R.; J. DeYoung and M. Doo-Hong, 2012. Coated
Alfalfa Seed, is it worth it?. (Disponible en: http://www. progressiveforage.com/forage-types/alfalfa/coatedalfalfa-seed-is-it-worth-it Consultado: 12/08/2016).

Murphy, W.M.; D.T. Dugdale and D.S. Ross, 1984. Fertilizer and lime-pellet requirements for seed of white clover used for improving permanent pastures. Grass and Forage Science. Vol. 39, pp. 281-284

Natural Resources Conservation Service (NRCS), 2010. Inoculating legumes. Illinois Agronomy Technical Note No. 20 (Disponible en: https://efotg.sc.egov.usda.gov/ references/public/IL/TechNotelL-20_Rev1.pdf. Consultado: 12/08/2016).

Parga, J., 1994. Consideraciones Técnicas para el establecimiento y manejo de la alfalfa. In: Torres y Bortolameolli. (Ed). Seminario utilización de alfalfa en la Décima Región. INIA Remehue. Osorno, Chile. Pp: 3-23.

Romero, L., 2008. Pasturas templadas. Implantación de pasturas. XXI Curso internacional de lechería para profesionales de América Latina. EEA INTA Rafaela. Pág. 40. (Disponible en: http://www.infortambo. com/admin/upload/arch/pasturastt.pdf. Consultado: 12/08/2016).

Shewmaker, G.E.; M.H. Hopwood and R.L. Roemer, 2002. Implication of seeding rates and seed coating with improved alfalfa varieties. In: Proceedings, Western Alfalfa and Forage Conference. University of California. (Disponible en: http://alfalfa.ucdavis. edu/\%2Bsymposium/proceedings/2002/02-185.pdf Consultado: 19/08/2016).

Talley, B., 2010. Advances in alfalfa seed coatings. In: Lacefield, G. y Forsythe, C. (Eds.) 30th Kentucky alfalfa conference proceedings. Universtity of Kentucky. (Disponible en: http://www.uky.edu/Ag/Forage/30th\%20 Kyalfproc\%20with\%20cover\%20plus.pdf . Consultado: 12/08/2016)

Talley, B., 2012. Status and new developments in seed coatings. (Disponible en: http://www. progressiveforage.com/forage-production/planting/ status-and-new-developments-in-seed-coatings. Consultado: 12/08/2016).

Twidwell, E.K. and D.J. Gallenberg, 2002. Use of seed coating and fungicide seed treatment in establishing Alfalfa. (Disponible en: http://openprairie.sdstate.edu/ cgi/viewcontent.cgi?article $=1301 \&$ context=extensi on_extra Consultado: 19/08/2016) 